\title{
HR ANALYTICS: A MODERN TOOL IN HR FOR PREDICTIVE DECISION MAKING
}

\author{
Dr. Abdul Quddus Mohammed \\ Assistant Professor in Business (HR), Higher Colleges of Technology \\ Abu Dhabi, United Arab Emirates
}

\begin{abstract}
Developments in Human Resources Management (HRM) are fast being integrated with corresponding changes in data and information processing, which are restructuring our environments. The domain of human resource analytics, which can be understood as a data and analytical thinking-centred approach to Human Resources Management, is fast becoming an indispensable part of organisational setups. The present study explores the existing literature in the field of HR analytics and their implications for predictive decision-making in organisations. This will also include critically reviewing the literature on the integration of HR analytics in organisational setups through the introduction of relevant IT infrastructure and provisions.
\end{abstract}

Keyword: HR analytics, predictive, decision-making, predictive modelling.

Cite this Article: Dr. Abdul Quddus Mohammed, HR Analytics: A Modern Tool in HR for Predictive Decision Making, Journal of Management, 6(3), 2019, pp. 51-63. http://www.iaeme.com/jom/issues.asp?JType=JOM\&VType=6\&IType=3

\section{INTRODUCTION}

In a competitive market scenario, it is imperative that an employee's potentials be harnessed to the best for organizational success. In such an environment, human resources remain one of the primary distinguishing factors for an organization that can be used for competitive growth in order to create necessary organizational value (Bharti, 2017). The optimum utilisation of the human resources capital that an organization possesses is an on-going process; consistent efforts in the direction will ensure that the human resources of an organization would remain an asset and not a liability. Human Resource Management must be undertaken taking into consideration the needs of the organization as a whole; it can be understood as a domain of study that is oriented towards exploring those practices and approaches, which can be implemented in the context of employees to achieve organizational goals (Armstrong and Taylor, 2014). However, for a Human Resources Management to be appropriately effective and help in making alterations and introductions that yield positive results or have profitable implications, it should be oriented towards gaining a deeper insight into behavioral particularities and characteristics of its employees. Stemming from the domain of Personnel Management, HRM is oriented towards identifying tools and measures, and relies on the basic principle that the employers and 
employees can work together and realize shared goals within the operative space of hierarchies and structured systems (Marchington, Wilkinson, Donnelly and Kynighou, 2016). In order to realise these goals, HRM includes a variety of established strategies and practices that have been proven to be effective and also the creation of new ones particular to organizational context.

Managerial tasks and decision-making on critical issues form an integral part of the work, which falls under the scope of the HRM of an organization. Decision-making has been identified as one of the most critical organizational processes including employee behavior, work performance, levels of motivation and the amount of stress levied on employees (Griffin and Moorland, 2011). It is critical that the nature of HRM practices implemented would be aligned and synchronized with larger expectations and guidelines for employee behavior and competitive goals. Keeping in perspective, the desired role behavior of an employee, that is, the requisite skills, knowledge dimensions and abilities, various competitive business strategies can be closely matched with organizational conditions in order to see development in critical areas, such as decision-making (Pereira, 2013).

Human resource analytics is a relatively novel intervention in the larger domain of HRM, and it refers to the use of statistical tools, measures and procedures, which can be used in employing and masking the most effectual decisions such as HRM strategies and practices. It is often referred as people analytics or talent analytics or workforce analytics ("People Analytics", n.d.). HR analytics can be understood as being more credible because it provides statistically valid data and evidence that can be used in the process of creating new strategies during the implementation of existing HR strategies and other measures. The possibilities for HRM offered by analytics have been realized by employers and organizations, but there remains an immense room for growth in the area and the study of the relevance of analytics within the various categories that fall under HRM.

The present study is directed towards exploring the existing literature about the relationship between Human Resource analytics and the role it can play in improving the existing range of managerial and HR-related tasks. The exploration of this literature will be instrumental in providing insight into to what extent people analytics is relevant in the domain of decisionmaking and the ways in which it can be adopted by organizations to expect good returns on investments made in the process. This will include critical examination of the steps in detail taken for the integration of HR analytics in the organizational structure; the processes employed, and the statistical tools used for data storing and the approach adopted while putting analytics to use for industrious decision-making. Hence, HR analytics can be understood as offering significant prospects and has a huge potential of improving the HR and Managerial decisions-making process that will be explored during the present study.

\section{RESEARCH AIMS AND OBJECTIVES}

The aim of the following research is to undertake an exploration of existing literature with the aim of understanding the relationship between human resource and analytics and understand the role it plays in the improvement of the existing range of managerial and HR related research. The present study is 52ealizati around the 52ealization of the following objectives-

1. To investigate and gain insight into the future of HR analytics if integrated into the company to assist managers in predictive decision-making based on statistical evidences and relevant HR analytical data and literature.

2. To examine the existing literature on the integration of HR analytics within organisations and evaluate the existing studies qualitatively and discuss the research gaps (if any). 
3. To examine the IT infrastructure and technological interventions, including those that affect the way data is mined stored and made in terms of the effective implementation of HR analytics and the need for them in order to be efficient in terms of data storing in order to be relevant for HR analytics

\section{LITERATURE REVIEW}

\subsection{Integration of HR Analytics within a company}

\subsubsection{The Concept of HR Analytics}

People are organizations unsurpassed assets, and effective way of gaining competitive advantage in a present volatile market environment and it is a big challenge for organizations to manage employees with diversified competencies and mapping their outputs in line with the organizational strategy. This requires creating, analysing and storing vast amount of data to support decision making. Management of human resources requires tools to enable managers to get insights into the patterns that emerge from various HR functions, which will help the organizations in filtering the star performers from the pool of huge employee database. The solution is offered by the implementation of analytics for the management of employee data scientifically and rationally and relating with the organizational outcomes. "HR Analytics" includes the use of statistical techniques, research design, and algorithms to evaluate employee data and translating results into evocative reports (Levenson 2005). The HR Analytics applies statistical models to get insights into employee data, patterns revealed by the data makes it possible to predict employee behavioural patterns like attrition rates, training costs, and employee contribution. This is also called as predictive analysis.

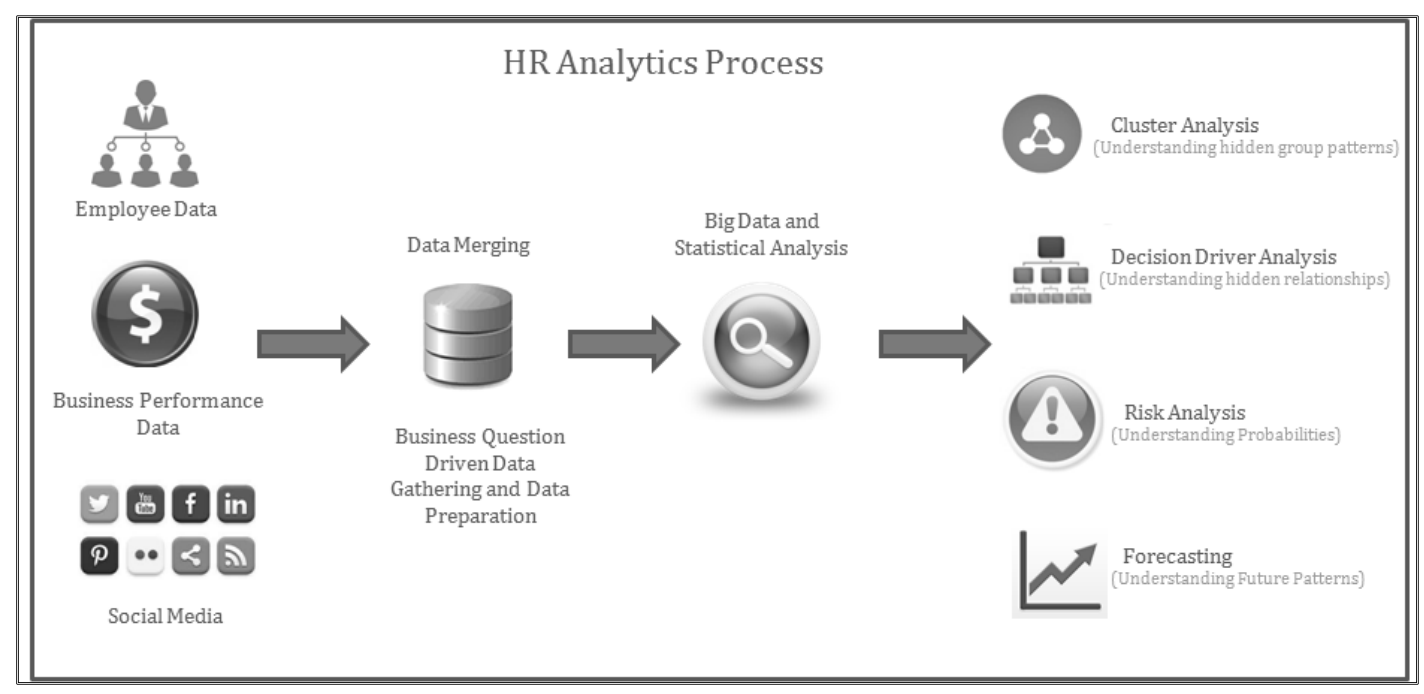

Figure 1 HR Analytics Process

A typical HR Analytics System collects employee data from HRIS (Human Resources Information System), business performance records, mobile applications and social media merges into a Data Warehouse, applies big data, statistical analysis and data mining techniques to provide understanding of hidden data patterns, relations, probabilities and forecasting. A Data Warehousing System deals with the data collection, analysis, and transformation and storing data on various databases.

HR analytics is a relatively novel intervention in the larger domain of Human Resource Management. It is also often referred to as people analytics or talent analytics or workforce analytics ("People Analytics," n.d.). HR analytics can be understood as being more credible 
because it provides statistically valid data and evidence that can be used in the process of creating new strategies and during the implementation of existing HR strategies and other measures. The possibilities for HRM offered by analytics have been realized by employers and organizations, but there remains immense room for growth in the area and the study of the relevance of analytics within the various categories that fall under Human Resource Management.

The effective HR Analytics will help the HR managers in performing HR functions such as forecasting the demand and supply of people, identifying suitable employments tests to suit applicant profiles, assessing training needs of employees, implementing pay for performance, and maintaining effective employee information to decide on rewards and managing employee discipline. Overall it helps the HR managers to make decisions based on data about recruitment, retention, training, rewards, career planning and organizational effectiveness and efficiency.

\subsubsection{Types of HR Analytics:}

Analytics can be categorised as descriptive, predictive and optimization analytics (Watson 2010, Narula 2015). Descriptive Analytics is a first level of analysis, includes understanding the historical data, behavior and outcomes, it only describes the relationship (Fitz-enz 2009). It involves the use of data visualization, adhoc reports, drilling-down, dashboards / score cards, SQL Queries. Turnover rates, Cost per hire and Absence Rates can be found out using descroptive analysis. The second level of analysis is Predictive Anaytics includes forecasting the futre behavior and outcomes based on the past data. It involves the use of Data Mining (correlation between data), decision trees, pattern recognition, forecasting, root-cause-analysis, and predictive modeling (what will happen next). Predictive modeling will help the HR managers in forecasting attrition rates, proabibility of employee success on job based on recruitment / selection methods used. The third level of analysis is Optimization Analytics, includes not only achieving the best outcomes by using limited resources. It involves using linear programming, simulations, creating mathematical modelling and implementing are used to find the best alternative training investment to achieve organizational effectiveness (Narula, 2015).

\subsubsection{Why HR Analytics}

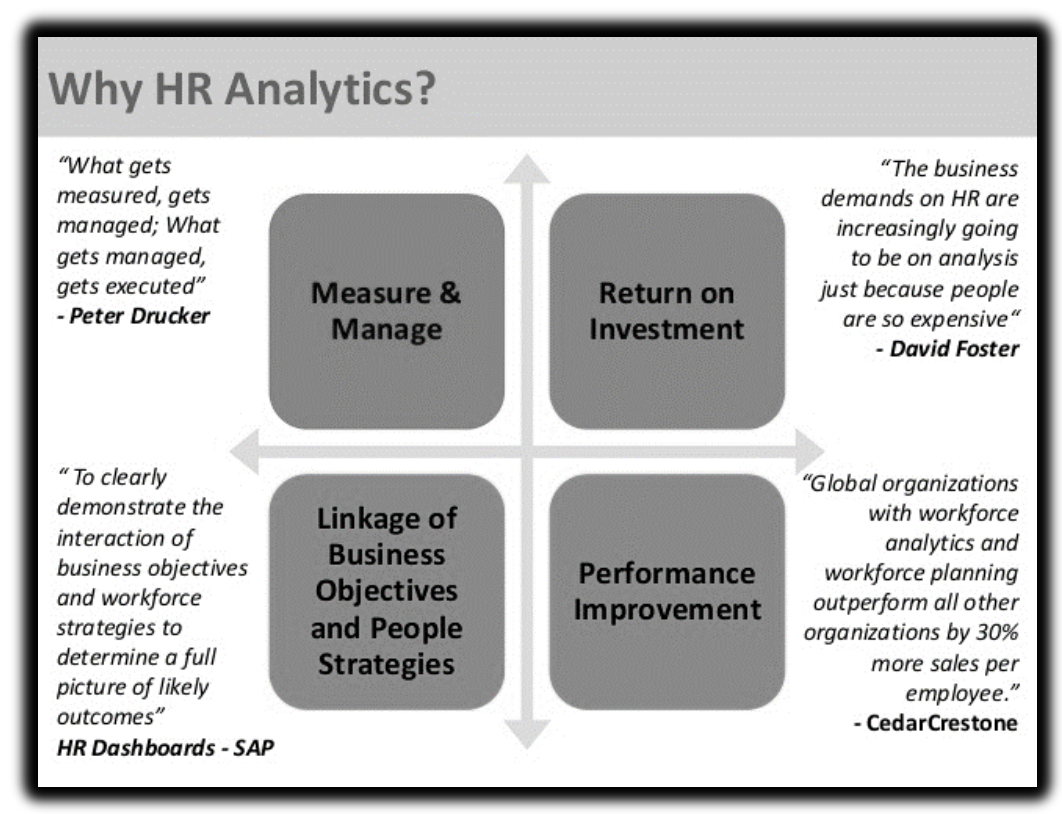

Figure 2 HR Analytics 


\subsubsection{HR Analytical Tools}

SAP, IBM, Oracle, Microsoft are vendors in Business Intelligence. BI software's have HR modules with BI and data analytics capabilities (Kapoor \& Sherif 2012). R-Studio is a data analysis and visualization software tool which can be applied for very large data sets, Python is a preferred programming language of all data scientists for statistical data analysis and visualization. Microsoft Excel is a traditionally a great data analysis tool to collect, analyse and transform data using formulas, pivot tables, scenario building and graph tools. Microsoft Power Business Intelligence software has power to extract data from various sources and makes easy for us to analyse, aggregate and visualization of data, the best part of this software is it (Erik Van Vulpen, n.d),

Mondore, Douthitt and Carson (2011) in their study on HR analytics provided a Six-step roadmap on how to include HR analytics so as to ensure that it aligns with HR strategies and desired organisational outcomes. The steps involved and described in the research include identifying the most significant outcomes; creating a cross-functional data plan; implementing adequate measures to assess critical outcomes; drafting and executing the plan; measuring, modifying it post-implementation and making other modifications (p. 23-24). The study also looked at how HR analytics can be used in order to identify and secure talent for an enterprise hence, making a plan for succession and talent for an organisation. The suggested plan for HR analytics outlined in the study is based on the implementation in certain organisations; hence it can be understood as providing a detailed insight through minor suggestions, such as making HR analytics available to individuals in leadership roles in an organisation.

Rasmussen and Ulrich's (2015) study examines the nature of the claim that HR analytics is said to bring additional value to managerial and HR decision-making by providing statisticalbacked and verified data. The study recommends that in order to prevent HR analytics from becoming yet another 'management fad', it should work towards altering the conventional approach adopted by HR and related endeavours in order to ensure that it is effective in terms of providing concrete and material outcomes. The study recommended that instead of employing the traditional HR-oriented "inside-out" approach, there is a need for shift towards the "outside-in" approach, which is implemented with an emphasis on concrete action. This suggestion for an altered approach could be implemented through the use of relevant technological interventions. Ulrich and Rasmussen's study presents two case studies on the implementation of HR analytics in business analytics and studies the positive implications it has in terms of optimising performance and managing the development of organisational talent. The study posits HR analytics as a judicious tool in order to enhance the real-world impact of HR in achieving business goals.

Levenson, in his 2011 study, advances the importance of analytics to HR practice in which it provides a comparative analysis of the cost benefit and impact analysis and the ROI. Building on his 2005 study, Levenson (2011) postulates that the use of analytics and metrics provide great potential for the improvement of the quality of decision making in human capital and HR issues in the firms. The study advances that the analytics in the HR function have been observed as a specialist skill preserved from the analytic specialist. Levenson's study points out that the metrics are important in the increasing the levels of insightful, analytically-based decision making in HR. Moreover, the study postulates that the metrics and analytics aids in much needed deep analysis in which he discusses the models such as labour markets, and organizational design, which all have elements of diagnostics and apply logic exercises. The study, thereby, suggests that the time, energy and resources of the HR practitioner should be on the application of good analytics broadly, which improve the diagnosis of the HR issues and provide a better orientation for the development of business models and actionable propositions. 
Additionally, another major suggestion by the study included the development of an HR analytics $\mathrm{COE}$ and ensuring a foundation based on HR analytics across the range of organisational tasks. Rasmussen and Ulrich's (2015) study examines the nature of the claim that HR analytics is said to bring additional value to managerial and HR decision-making by providing statistical backed and verified data. The study recommends that to prevent HR analytics from becoming yet another 'management fad', it should work towards altering the conventional approach adopted by HR and related endeavours to ensure that it is effective concerning providing concrete and material outcomes. The study recommended that the instead of employing the traditional HR oriented "inside-out" approach, there is a need for shift towards the "outside-in" approach that is implemented with an emphasis on concrete action. This suggestion for an altered approach could be implemented using relevant technological interventions. Ulrich and Rasmussen's study presents two case studies on the implementation of HR analytics in business analytics and studies the positive implications this has regarding optimizing performance and in managing the development of organisational talent. The study posits HR analytics as a judicious tool to enhance the real-world impact of HR regarding achieving business goals.

It is also important to consider how HR analytics to be used in line with the HR strategies and desired organizational outcomes, this was demonstrated by Mondore, Douthitt and Carson (2011) in their study on HR analytics provided a six-step roadmap on how to include HR analytics to ensure that it aligns with HR strategies and the desired organisational outcomes. The steps involved described in the research include identifying the most significant outcomes; creating a cross-functional data plan; implementing adequate measures to assess critical outcomes; drafting and executing the plan; measuring, modifying it post-implementation and making other modifications. The study also looked at how HR analytics can be used to identify and secure talent for the enterprise hence planning for succession and talent planning for the organization. The suggested plan for HR analytics outlined in the study is based on the implementation in certain organizations hence can be understood as providing detailed insight through minor suggestions such as making HR analytics available to individuals in leadership roles in the organization.

\subsection{IT infrastructure and centralised data storing}

The development of HR analytical systems has been accelerated by technology, which is rapidly consolidating the analytics landscape. This section will review literature pertaining to the use of technology in developing the scope of HR analytics in decision-making.

Fairhurst's study (2014) defined the nature of the steps that need to be taken regarding incorporating a data-driven architecture into HR analytics, to assimilate analytics with HRM. The study narrowed down five steps involved it the process, which include the conversion of business concerns into data interpretation questions, i.e. ones that can be answered through data. The other steps involved in the process include making necessary provisions for systemizing and storing the data acquired; the interpretation of data through relevant statistical tools and measures; further analysis of the data using statistics, machine learning and neural networking; and the presentation of the outcomes or findings to the concerned enterprise or organization in a coherent format. Angrave, Charlwood, Kirkpatrick, Lawrence and Stuart's (2016) study looked at the veracity of the transformative claims touted by HR analytics, which is fast becoming one of the imperatives for reworking organisational outcomes. The arguments presented by the study is a cautionary one, which seeks to look at the implementation of analytical thinking in HR analytics. In the domain of big data studies, Angrave et al. have reviewed the need for analytics related measures to be rooted in and based on metrics that simultaneously rely on advanced statistical analysis and on experiments carried out. The 
recommendation for improving the existing model is based on the inclusion of HR related variables in the creation of analytics models but at the same time the study cautions against the unchecked inclusion of solutions provided by analytics software and tools. King's (2016) study examined the role of data analytics in HRM through a case study and a critical review. According to the study the developments in the field have also fueled a degree of uncertainty about the proficiency of HR professionals in employing the data or information accessible to them in order to generate productive results. The study reviewed literature that is both for and looks at the drawbacks of human resource analytics and argued for the involvement of academic discourse in the inclusion of analytics and related practices. The use of case studies in the research provides practical advice through the study, with the emphasis on a more applied and action-oriented approach. Hence the study identified certain targets for analytics, recommended practices such as outsourcing, and ensuring that the available data was trustworthy and within reach and the development of models to put to use the available data.

Jasmit Kaur and Alexis A. Fink (2017) based 22 interviews at 16 companies found that most of the companies (94\%) are using $\mathrm{R}$ Studio is used for the data analysis and visualization, followed by Tableau (83\%) which is a popular visualization and dashboard creation tool ,some companies are also using Python for data analysis, data cleaning, data management and machine learning SPSS and Excel are also used by some companies as traditional and trust worthy tools for business analytics professionals. Some new technology tools are permitting Artificial Intelligence are IBM's Watson Talent, Intel's Saffron, HireVue's video analytics platform. For surveys Qualtrics, SurveyGizmo, Sirota are used and for business intelligence and analytics Cognos Visier are used. These list of new technology tools confirms that the HR analytics is still at experimentation stage and there is long way to go in the future.

\subsection{Predictive Decision Making}

Predictive analytics is applied to make forecasts about unknown events using statistical data analysis, modelling, machine learning, data mining and AI (Artificial Intelligence) to analyse historical and current data to predict future data. Predictive analysis in HR is rapidly growing and demanded area. HR predictive analytics is being used by HR managers in organizations to predict human behaviour and optimize performances and produce a better return on investment for organizations through decision making based on predictive analysis tools. This section will review relevant literature to understand the process of HR predictive analytics.

Rich, Lepine and Crawford's (2010) study theorised that employee engagement is a more significant marker affecting employee performance as compared with other factors that reflect relatively smaller aspects of an employee's personality. Their study was based on the evaluation of 245 firefighters with their supervisors in order to reach the desired conclusions about

engagement. According to the study, engagement mediates relationships between critical parameters like the performance at tasks and organisational behaviour exhibited by employees. Other parameters included mediators include involvement, satisfaction with the nature of work, the innate dedication of the employee towards the work were found to have significantly less effects when compared to the parameter of engagement.

Puhakainen and Siponen's (2010) study evaluated the need for employee training with respect to complying with the information system security policies of an organisation. According to the study, establishing the significance of the need for such training is in the interest of the organisation. The study suggested the implementation of two models as training programs in IS security policies, the primary program included training through an action research-based plan. The action research-based plan drew from theoretical contributions and examined their practical feasibility to creating awareness about IS security policies. 
Fitz-enz and Mattox's (2014) study comprehensively investigated the role of predictive analytics in the study looked at the three levels that analytics is divided in, namely predictive, descriptive and prescriptive analysis and the various techniques that fall under them. The study looked at the complete range of steps involved in the process beginning from creating an analytic value chain; to the creation of analytic models, the varied processes involved in turning data into information; along with certain examples of predictive statistics and predictive analytics in action. The research study also explicitly cites examples from analytics that demonstrate the use of predictive modeling. This involves the setting up of models that demonstrate that how raw data can be used to gain insight and to generate inferences for instance, exit interviews carried out by employers can be an important measure used to understand the reasons behind why employees choose to leave and hence help the organization in talent retention.

Laszlo Bock (2015) the Senior Vice President of HR at Google writes in his book Work Rules, argues that Google uses statistics in people management the interview questions are fully automated, perfectly tweaked based on the profile of the candidates to find the best suitable candidates. Google also estimates the probability of employees resigning from the company by applying HR predictive analysis that the employees who do not get promotion in first four years are likely to leave the company. Kluemper, Rosen \& Mossholder (2012) discovered the possibility to predict the employee job performance and behavior based o IQ, personality tests, structured interviews and there is correlation between these tests and the Facebook profile of the candidate, which could be used as an supplementary instrument of employee selection after considering legal and ethical issues. Best Buy applies HR analytics in predicting the store performance based on employee engagement practices for every quarter, it predicted that a 0.1 percent increase in employee engagement resulted in increase of store income to $\$ 100,000$ (Erik Van Vulpen n.d). In 2011 Hewlett-Packard (HP) applied HR data analytics to predict the number of employee turnover that they called "Flight Risk Score". They found that higher pay and the promotions and better performance ratings are negatively correlated, someone who received promotion but did not receive pay raise is likely to leave job. HP created a dashboard for all its HR managers with important matrices for all employee information. Companies can save millions of dollars by applying predictive HR analysis by predicting how the employees behave but also their contribution in organizational performance.

Ballinger, Cross and Holtom in their 2016 study examined the influence of the network structure as a predictor for voluntary turnover. The research focused on the employee's social relationships as a resource that influences the rate of labour capital in the firm. according to the study, the network reputation which serves as an access to well-connected people is an influential factor in determining the turnover within the organization. Ballinger, Cross and Holtom (2016) observe that the brokerage serves as a moderating factor in which it serves as a gain in terms of the advancement, the generation of ideas and the performance. The primary inference to the employee turnover that can be drawn from the study is that that employees who had a more established social network have more brokerage and reputation resources and are more likely to leave the firms. Mishra, Lama and Pal's (2016) study examined the role of the domain of predictive analytics within HR analytics, according to the study HR predictive analytics has wide-ranging effects regarding the all areas related to human resource management through the identification and proposal of certain metrics that can be used for predictive modelling. It helps organizations in reducing implementation costs due to HR related interventions, and to optimize business performance and see higher levels of employee involvement. The study helps in explaining the connection between factors like low talent retention and high rate of attrition. According to the study HR predictive analytics "is rapidly changing and growing technology which has potential to achieve $100 \%$ accuracy in decision making for HR. Till 2020," (p. 34). 
Reddy and Lakshmikeerthi's (2017) in their study found that HR analytics poses a way of being an important tool that can help assimilate the data gathered to describe, analyze, predict and optimize the potential of an organization's employees. The study also narrowed down certain factors like institutional mechanisms, competitive mechanisms, configuration and organization architecture, that are instrumental in effecting HR analytics.

Jasmit Kaur and Alexis A. Fink (2017) based 22 interviews at 16 companies found the companies are using HR analytics for building predictive models for hiring, retention, attrition and designing employee benefits based on employee demographics and attrition patterns. Companies are using HR analytics for employee engagement through satisfaction surveys, building staffing plans based on data collected from the HR departmental vacancy positions, talent movement (transfers, promotions) and labour market data, the employee feedback on good or worst management practices are collected to enable managers understand their styles and needs for improvement. The measurement of effectiveness of training programs is also an important application of HR analytics.

\subsection{Data Governance issues in HR Analytics}

Data Governance is use of formal procedures to make sure that the quality data is collected and to be used ethical and legal way. The area of HR Analytics has potential of issues related to ethics and legality as it is using modern technologies to source, analyse large volumes of data to facilitate managerial decision making. Toyama, K. (2015) recommended that first sin of Artificial Intelligence is to allow computer systems (machines) to make ethically sensitive decisions. And recommended that the HR analytics can be input to human decision makers. Predictive models can be useful to alert decisionmakers but not to make or arrive at the decisions. As the decisions should not violate important labour laws such as discrimination, disabilities. There is a danger of employee confidentiality, trust and privacy because of HR data analytics and using clean data for decision making.

\section{DISCUSSION}

In the literature reviewed on the integration of HR analytics within the organisation, the strategic plan outlined by Mondore, Douthitt and Carson's study does not come with sufficient reviews or case studies that could provide sufficient evidence of its real-world applicability. As such the model posited by the study cannot be assumed to be universally applicable and must be contextualised according to a particular organisation and its specifications. Rasmussen and Ulrich's (2015) study is based on the notion of revolutionising the approach adopted by HR organisations in terms of aligning analytics with the business goals of the study, but hasn't seen adequate implementation in order to make evident the extent to which HR analytics reflected returns in terms of helping in attaining organisational goals. Levenson's study looked at the various tools and statistical procedures that fall in the domain of HR analytics in order to surmise their particular utility for HR analytics. The study also emphasised the development of business models and concrete strategies instead of the regular implementation and deployment of resources for data mining. However, the research doesn't identify particular time frames and resources needed for the development of recommended HR analytics COE and for drafting models required for the implementation of HR analytics.

In the literature reviewed under the section entitled IT Infrastructure and Centralised Data Storing, Angrave et al. (2016) provides statistically backed evidence and data gathered from analytical statistics and the inclusion of HR-related variables in the information. While the study endorses the formulation of an analytical model that is tried and tested, and such a practicable approach suggested by the study is beyond the scope of a theoretical analysis and can only be undertaken in an organisational setup. Additionally, the study also points out that data analytics 
results and recommendations may not be without error, and hence cannot be relied on blindly. A week's study claims that the inclusion of online and web-based HRIS will simplify the process of acquiring HR variable dependent data, making it primarily machine-dependent and reduce the need for human resources; however, at this stage, the feasibility of the HRIS systems in terms of their cost and other factors related to their inclusion can only be approximated. Other impediments to the introduction of HRIS may crop up, such as expensive technical components, and the need for personnel for monitoring them. The optimistic claims made by the study do not take this into consideration. Fairhurst's (2014) study presents a model for including a datadriven architecture into an organisational setup, so as to manage the data acquired for HR analytics. Yet, the model presented by the study remains a cursory formulation and does not provide an in-depth explanation of each of these steps. As such the practical feasibility of the model remains limited and the task of incorporating it in an organisational framework would require considerable effort.

The claims of Mishra, Lama and Pal's study about Predictive Decision-Making need to be supplemented with adequate case studies done in organisations, in order to legitimise those postulations relating to the cost sustainability prospects offered by HR analytics. Additionally, the estimates for future growth in the field posited by the study remain at face value, and the feasibility of these claims needs to be studied in the context of real-world enterprises. Fitz-enz and Mattox's study, on the other hand, provides a more comprehensive roadmap for including analytics into HRM with detailed insight into the nature of the shift in approach required in order to adopt a predictive model, which favours decision-making for the sake of human resource management. by Ballinger, Cross and Holtom 's and Rich's study are useful in terms of offering parameters around which predictive decision-making parameters like performance and employee turnover can be based. But the suggestions offered by studies were in isolation; without an action plan or a model for their implementation. Puhakainen and Siponen's study for the implementation of a training program similarly suffers from the lack of an adequate model to be tested for practical feasibility in organisations.

\section{HR ANALYTICS AND PREDICTIVE DECISION-MAKING MODEL}

Post the review of a relevant existing literature on HR analytics and predictive decision-making, the following model was designed considering the relevance of effective decision-making for organisational success. The proposed model is based on the literature reviewed earlier.

The outlined model, Figure 3 is based on the studies surveyed by Ballinger, Cross and Holtom (2016) whose study posits how data acquired about the network structure of the employees can serve as a predictor for employee turnover rates. Similarly, the proposed model looks at how data acquired through various parameters can affect employee performance, as outlined in Rich et al' (2010) study, and can be evaluated using analytics tools. As per the literature reviewed earlier, Puhakainen and Siponen's study evaluated the need for employees to be trained in the information systems security policies of the organisation in order to ensure that they comply with the prescribed policies. In the context of the present study, the required training-related interventions that need to be made in this regard can be understood through data gathered on the existing levels of training and awareness among employees. Once such information has been acquired, it can be analyzed through analytics tools in order to lead to conclusions about predictive decisions that need to be made with respect to training requirements and provisions for employees. The model presents a visual representation of how analytics-based interventions can be used for predictive decision-making about various parameters that are pivotal to organizational operation. 


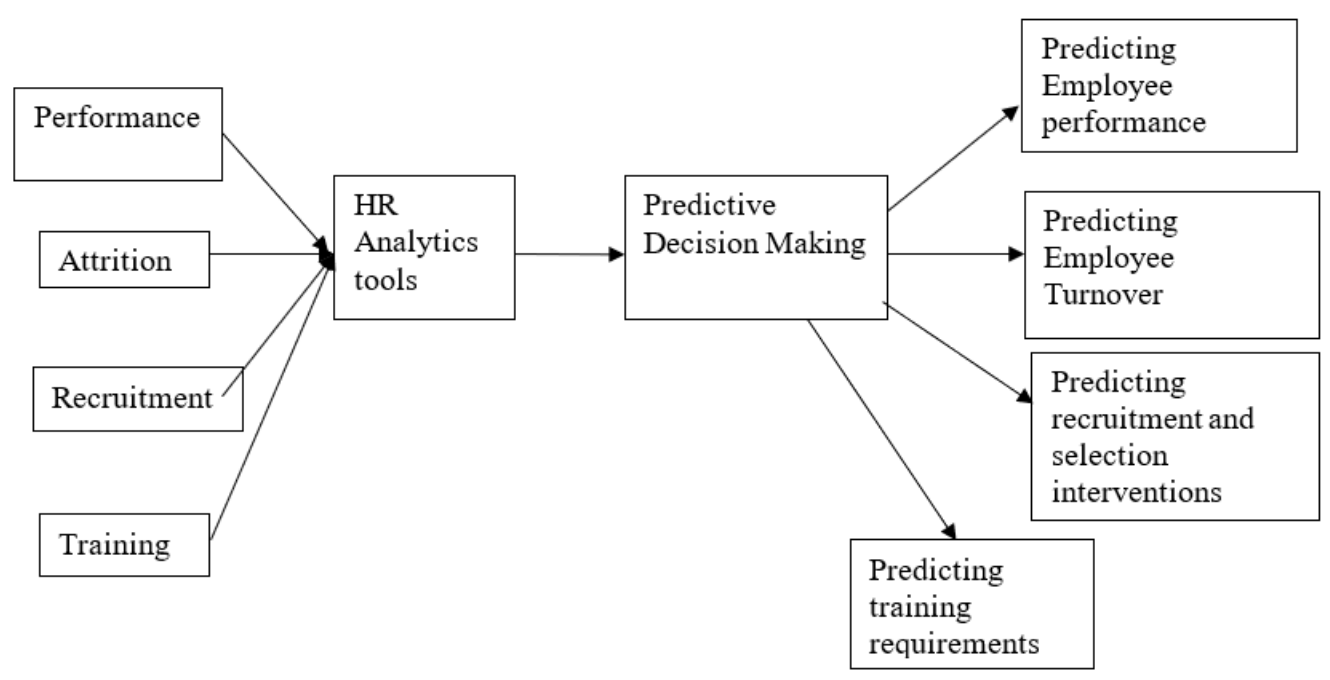

Figure 3 HR Analytics and Predictive Decision-making model

\section{RESULTS AND CONCLUSIONS}

The present research aimed for an exploration of existing literature with the aim of understanding the relationship between human resource and analytics and understand the role it plays in the improvement of the existing range of managerial and HR related research. The study's focus was on investigating on the future of HR analytics if integrated into the company to assist managers in predictive decision-making based on statistical evidences and relevant HR analytical data and literature. Also, the focus was on examining the IT infrastructure and technological interventions, including those that affect the way data is mined stored and made in terms of the effective implementation of HR analytics and the need for them in order to be efficient in terms of data storing in order to be relevant for HR analytics.

A significant conclusion drawn from the review of literature on the integration of HR analytics in the domain of human resource management makes it clear that while suggestions for theoretical models that could be adopted for the hassle-free integration exist, there is a lack of literature that reviews the real-world applicability of these models and has reviewed the success (or failure) of these models or strategies when implemented in the context of organisations.

Exploration of the literature related to predictive behaviour point out that the data analytics in the field can help in identifying specific parameters that can aid both HRM and HRD in an organisation. Predictive modelling, similar to the model outlined in the present study, can be used to ensure that raw data helps in drawing important inferences and genuine insights that aid organisational growth. However, the existing literature resources could be further enhanced in terms of studying case studies of predictive models to accurately gauge the relevance and feasibility of the models created for specific industry sectors. This is significant because predictive decision-making and modelling cannot be homogenously used across industry types and enterprises as they will vary according to various parameters such as type of enterprise, the industry it is functional in, the number of employees and amount of resources it can expend for HR analytics.

\section{CONTRIBUTION TO KNOWLEDGE}

This paper contributes greatly to the field of human resource management in which the information explores the range of possibilities that HR analytics opens up for tasks in HR and administration. The findings point to possible success of analytics-based intervention and how 
it impacts the predictive decision making on parameters which are pivotal to the operation within the organization. Further insights and significant conclusions, for the scope of the largescale implementation of HR analytics and its implications for future, can be drawn from the review of literature carried out. The conclusions drawn can also be useful for comprehending the range of possibilities that HR analytics opens for tasks such as predictive decision-making and the limitations of these posited tasks.

\section{RESEARCH LIMITATIONS}

The main limitations of the following research predominantly centre on the availability of literature on the issue. There is an existing gap in which the existing literature on predictive modelling and organizational studies is limited in range. Hence, there is a need for existing literature to be sufficiently nuanced and to be based on case studies of predictive modelling and organisational studies so as to ensure that models and roadmaps being endorsed are tried, tested and fail-safe. Due to this, the present study was limited in the range of literature available for review.

\section{SUGGESTIONS FOR FUTURE RESEARCH}

Future research should consider focussing on case studies of predictive modelling and organisational studies in an effort to identify the models and roadmaps from different organizations which have been tested, tried and successful. The research should also focus on a more quantitative approach to the research with the aim of observing predictive modelling as an aspect of HR analytics on an organizational setting.

\section{REFERENCES}

[1] Angrave, D., Charlwood, A., Kirkpatrick, I., Lawrence, M., \& Stuart, M. 2016. HR and analytics: why HR are set to fail the big data challenge. Human Resource Management Journal, 26(1), pp1-11.

[2] Anon. People analytics (HR analytics). Retrieved February 26, 2018, from http://searchhrsoftware.techtarget.com/definition/human-resources-analytics-talentanalytics

[3] Armstrong, M., \& Taylor, S. 2014. Armstrong's handbook of human resource management practice. Kogan Page Publishers.

[4] Bharti, A. 2017. Human resource analytics. South Asian Journal of Marketing \& Management Research, 7(5), pp 68-77.

[5] Fairhurst, P. 2014. Big data and HR analytics. Institute for Employment studies.

[6] Ballinger, G. A., Cross, R., \& Holtom, B. C. 2016. The right friends in the right places: Understanding network structure as a predictor of voluntary turnover. Journal of Applied Psychology, 101(4), pp 535-548. doi:10.1037/ap10000061

[7] Fitz-enz, J., \& Mattox, J. R. 2014. Predictive analytics for human resources. Hoboken, NJ: Wiley.

[8] Griffin, R. W., \& Moorhead, G. 2011. Organizational behavior. Cengage Learning

[9] King, K. G. 2016. Data Analytics in Human Resources: A Case Study and Critical Review. Human Resource Development Review, 15(4).

[10] Levenson, A. 2011. Using targeted analytics to improve talent decisions. People and Strategy, 34(2), pp 34.

[11] Marchington, M., Wilkinson, A., Donnelly, R., \& Kynighou, A. 2016. Human resource management at work. Kogan Page Publishers. 
[12] Mishra, S. N., Lama, R., \& Pal, Y. 2016. Human Resource Predictive Analytics (HRPA) For HR Management In Organisations. INTERNATIONAL JOURNAL OF SCIENTIFIC \& TECHNOLOGY RESEARCH VOLUME, 5(5). Retrieved February 23, 2018.

[13] Mondore, S., Douthitt, S., \& Carson, M. 2011. Maximizing the impact and effectiveness of HR analytics to drive business outcomes. People and Strategy, 34(2), pp 20.

[14] Pereira, V. E. 2013. A Longitudinal Case-Study Examination of HRM Practices in HighPerforming Work Organisations in the Indian HRO/BPO Industry (Unpublished master's thesis). University of Portsmouth. Retrieved February 22, 2018, from https://researchportal.port.ac.uk/portal/files/6033330/Vijay_Phd_Oct_2013_Revised.pdf

[15] Puhakainen, P., \& Siponen, M. 2010. Improving Employees' Compliance Through Information Systems Security Training: An Action Research Study. MIS Quarterly, 34(4), pp 757-778. doi: $10.2307 / 25750704$

[16] Rasmussen, T., \& Ulrich, D. 2015. Learning from practice: how HR analytics avoids being a management fad. Organizational Dynamics, 44(3), pp 236-242.

[17] Reddy, P. R., \& Lakshmikeerthi, P. 2017. 'HR Analytics' - An Effective Evidence Based HRM Tool. International Journal of Business and Management Invention, 6(7) 